\title{
EFEITOS DO TREINAMENTO EM JOGOS REDUZIDOS COM INFERIORIDADE NUMÉRICA NO FUTEBOL
}

\author{
EFFECTS OF TRAINING IN REDUCED MATCHES WITH NUMERICAL INFERIORITY IN FOOTBALL
}

EFECTOS DEL ENTRENAMIENTO EN PARTIDOS REDUCIDOS CON INFERIORIDAD NUMÉRICA EN EL FÚTBOL

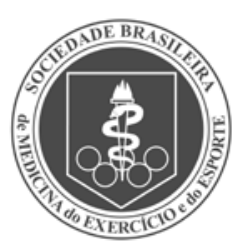

Artigo Original

Original Article Artículo Original
Victor Hugo de Siqueira Montalvão' (Profissional de Educação Física)

Guilherme Pascoal Mereu' (Profissional de Educação Física)

Alexandre de Souza e Silva' (Profissional de Educação Física)

Fábio Vieira Lacerda' (Nutricionista)

Jasiele Aparecida de Oliveira Silva' (Psicóloga)

Ronaldo Júlio Baganha’ (Profissional de Educação Física)

Luís Henrique Sales Oliveira' (Fisioterapeuta)

1. Centro Universitário de Itajubá, Fundação de Ensino e Pesquisa de Itajubá (FEPI), Itajubá, MG, Brasil.

\section{Correspondência:}

Alexandre de Souza e Silva. Av. Dr. Antônio Braga Filho, 687, Varginha, Itajubá, MG, Brasil. 37501-002.

alexprofms@yahoo.com.br

\section{RESUMO}

Introdução: Os jogos reduzidos são altamente eficientes, pois permitem a simulação de movimentos que ocorrem durante uma partida e geram melhor resposta fisiológica, perceptiva e técnico-tática. Objetivo: Comparar os efeitos do treinamento de jogos reduzidos com inferioridade numérica 3 vs. 4 e 4 vs. 5 sobre a variação da frequência cardíaca, percepção subjetiva de esforço e teste de atenção concentrada em atletas de futebol. Métodos: A amostra foi composta por 10 indivíduos do sexo masculino, com idade entre 17 e 24 anos. Para análise da frequência cardíaca média e máxima durante os jogos com campo reduzido e o coletivo foram utilizados monitores de frequência cardíaca e, para determinar a percepção subjetiva de esforço, utilizou-se de uma adaptação da escala de Borg. Também foi usado o teste de atenção concentrada. Resultados: Os resultados demonstram que os métodos de treino apresentam diferenças no teste de atenção concentrada $(p<0,004)$. A frequência cardíaca média $(p<0,121)$, máxima $(p<0,404)$ e a percepção de esforço $(p<0,639)$ não apresentam diferenças entre os métodos de treinamento. Conclusão: Concluímos que os jogos reduzidos com inferioridade numérica melhoram os resultados do teste de atenção concentrada.

Descritores: futebol; educação física e treinamento; frequência cardíaca; percepção; atenção.

\section{ABSTRACT}

Introduction: Reduced games are highly efficient because they allow the simulation of movements that occur during a match and generate better physiological, perceptual and technical-tactical response. Objective: To compare the effects of reduced training games with numerical inferiority 3 vs. 4 and 4 vs. 5 on heart rate variation, subjective perception of exertion and focused attention test in soccer players. Methods: The sample consisted of 10 males, aged between 17 and 24 years. For the analysis of the mean and maximum heart rate during the games with reduced field and players, heart rate monitors were used, and to determine the subjective perception of effort, an adaptation of the Borg scale was used. The concentrated attention test was also used.. Results: The results show that the training methods differ in the concentrated attention test $(p<0.004)$. Mean heart rate $(p<0.121)$, maximal $(p<0.404)$, and perceived exertion $(p<0.639)$ did not show differences between training methods. Conclusion: We conclude that the reduced games with numerical inferiority improve the results of the concentrated attention test.

Keywords: soccer; physical education and training; heart rate; perception; attention.

\section{RESUMEN}

Introducción: Los juegos reducidos son altamente eficientes porque permiten la simulación de movimientos que ocurren durante un partido y generan una mejor respuesta fisiológica, perceptual y técnico-táctica. Objetivo: Comparar los efectos de la reducción de los juegos de entrenamiento con inferioridad numérica 3 vs. 4 y 4 vs. 5 en la variación de la frecuencia cardiaca, la percepción subjetiva del esfuerzo y la atención enfocada en los jugadores de fútbol. Métodos: La muestra consistió en 10 varones, con edades comprendidas entre 17 y 24 años. Para analizar la frecuencia cardiaca media y máxima durante los juegos con campo reducido y menos jugadores se utilizaron monitores de frecuencia cardiaca y para determinar la percepción subjetiva del esfuerzo se utilizó una adaptación de la escala de Borg. También se utilizó la prueba de la atención concentrada. Resultados: Los resultados muestran que los métodos de entrenamiento difieren en la prueba de atención concentrada ( $p<0,004)$. La frecuencia cardiaca media $(p<0,121)$, máxima $(p<0,404)$ yel esfuerzo percibido $(p<0,639)$ no mostraron diferencias entre los métodos deentrenamiento. Conclusión: Se concluye que los juegos reducidos con inferioridad numérica mejoran los resultados de la prueba de atención concentrada.

Descriptores: fútbol; educación y entrenamiento físico; frecuencia cardiaca; percepción; atención.

\section{INTRODUÇÃO}

O futebol é um esporte altamente imprevisível e complexo uma vez que compreende a interação entre fatores técnicos, táticos, físicos e psicológicos ${ }^{1}$. Assim, para atender a estes aspectos, fez-se necessário buscar novos métodos de treinamento e dentre estes, os jogos reduzidos tem se mostrado altamente eficientes ${ }^{2}$. Os jogos reduzidos permitem a realização de ações motoras que ocorrem durante uma partida, o que possibilita uma melhor resposta fisiológica, perceptiva e técnico-tática ${ }^{3-6}$. Além disso, os 
jogos reduzidos favorecem uma melhora no condicionamento aeróbico, por meio de adaptações no sistema cardiorrespiratório e metabólico em função de sua alta intensidade ${ }^{7-9}$. As variáveis cardiorrespiratórias são mais responsivas a exercícios de elevada intensidade, na condição de inferioridade numérica, observada em jogos reduzidos ${ }^{10}$. A frequência cardíaca é uma variável utilizada como indicador da intensidade do exercício, assim como a percepção subjetiva de esforço ${ }^{11,12}$. Tem sido sugerido que o tempo e a intensidade da atividade podem afetar algumas variáveis psicológicas como a atenção concentrada ${ }^{13}$. No entanto, poucos estudos são encontrados na literatura referindo-se aos treinamentos com inferioridade numérica e os efeitos deste sobre o teste de atenção concentrada, frequência cardíaca e percepção subjetiva de esforço, o que torna esta pesquisa relevante, uma vez que, se confirmada a hipótese de que os jogos reduzidos em inferioridade numérica potencializam o estresse cardiovascular em relação ao jogo coletivo e que este influencia na percepção subjetiva de esforço e variáveis atencionais, os treinadores poderão utilizar este método para potencializar as adaptações cardiovasculares melhorando o condicionamento físico, o que poderá influenciar na variável de atenção concentrada, melhorando assim o desempenho dos atletas durante uma partida de futebol. O objetivo do estudo foi comparar os efeitos do treinamento de jogos reduzidos com inferioridade numérica 3 vs. 4 e 4 vs. 5 sobre a variação da frequência cardíaca, percepção subjetiva de esforço e teste de atenção concentrada em atletas de futebol.

\section{MÉTODOS}

A pesquisa se caracteriza por ser primaria e quantitativa, constituída de 10 atletas de nível estadual, idade entre 17 e 24 anos do gênero masculino e pertencentes à categoria juvenil de uma equipe de futebol de Minas Gerais, Brasil. Todos os atletas praticavam futebol à no mínimo três anos, com frequência de quatro dias na semana e duração de cada sessão de duas horas. Os grupos foram divididos em 3 vs. 4 jogadores, 4 vs. 5 jogadores e o coletivo (grupo controle). Todos os atletas participaram aleatoriamente e de maneira cruzada de todos os três grupos. Os responsáveis pelos participantes do estudo foram informados sobre os procedimentos de realização e assinaram o Termo e Consentimento Livre e Esclarecido da pesquisa. Foram inclusos no estudo atletas que não apresentavam histórico de doenças cardiovasculares, hipertensão arterial sistêmica e lesões osteomusculares, comprovado por meio de atestado médico. Este estudo foi aprovado pelo Comitê de Ética e Pesquisa do Centro Universitário de Itajubá/Brasil, sob o protocolo no 197.118 e está de acordo com a Resolução n 466/12 do Conselho Nacional de Saúde.

\section{Instrumentos}

A massa, altura e o Índice de Massa Corporal (IMC) dos atletas foi verificada por uma balança da marca Welmy ${ }^{\circledast}$ (Brasil) com capacidade até $180 \mathrm{~kg}$ e frações de 100g, tendo um estadiômetro acoplado ${ }^{14}$. Para a circunferência foi usada uma fita metálica da marca Cescorf ${ }^{\circledast}$ (Brasil), com $2 \mathrm{~m}$ de comprimento e $6 \mathrm{~mm}$ de largura ${ }^{14}$. Também foi utilizado um compasso científico da marca Cescorf ${ }^{\circledR}$ (Brasil), com precisão de leitura de 0,1 mm e pressão constante de $10 \mathrm{G} / \mathrm{mm}^{2}$ para analisar as dobras cutâneas e o percentual de gordura ${ }^{14,15}$.

Para monitorar a frequência cardíaca média e máxima foram utilizados monitores cardíacos da marca Polar ElectroOy ${ }^{\circledR}$, Vantage (Finland) ${ }^{16-18}$. A percepção subjetiva de esforço foi verificada por meio de uma adaptação da escala de Borg de esforço percebido, sugerida por Pasquarelli et al. ${ }^{9}$, que indica a resposta subjetiva de cada indivíduo, numa escala de intensidade de esforço, após uma sessão de treinamento ${ }^{19}$. Foi utilizado o teste de atenção concentrada, o qual consiste em 21 linhas contendo diversos símbolos, dentre os quais apenas sete em cada linha devem ser marcados. No início da folha há um pequeno retângulo mostrando os símbolos que devem ser marcados, para que não seja necessário decorá-los. Existe também um tempo de aplicação de 5 minutos, fazendo com que o teste avalie a capacidade dos indivíduos em focar na atividade, pois é avaliado a atenção e o controle cognitivo e mental sob situações de pressão ${ }^{13,20}$.

Os atletas na avaliação antropométrica ficaram com roupas leves e sem sapatos. Para o cálculo do IMC foi utilizado a seguinte fórmula: peso/altura ${ }^{2}\left(\mathrm{~kg} / \mathrm{m}^{2}\right)^{14}$. Para medir as circunferências foram utilizados os pontos anatômicos respeitando o protocolo de medição da equação original ${ }^{14}$. A relação cintura e quadril (RCQ) foi calculada dividindo-se a circunferência da cintura pela circunferência do quadril ${ }^{14}$. O percentual de gordura foi calculado utilizando sete dobras (subescapular, peitoral, axilar-média, tricipital, supra-ilíaca, abdominal e coxa). O protocolo utilizado foi o de Pollock e Jackson ${ }^{15}$

Para análise das variáveis dependentes foram consideradas a frequência média e máxima, obtida por meio do monitor cardíaco, durante as sessões de treino de jogos reduzidos e coletivo ${ }^{16-18}$. A percepção de esforço ${ }^{3,19}$ e a atenção concentrada foram avaliadas imediatamente após as sessões de treino ${ }^{13}$. Para a resposta subjetiva de esforço os indivíduos foram orientados antes da sessão de treino, e logo após o jogo, foram coletadas as respostas de cada atleta ${ }^{3,19}$. No teste de atenção concentrada foi feita uma explicação geral para os atletas, em seguida, foi realizada a fase de treino do teste e por fim o teste propriamente dito ${ }^{13,20}$

Os dados foram coletados durante as sessões de treinamento diário, onde todos os atletas participaram em dias diferentes de maneira aleatória de situações de jogos reduzido com 3 vs. 4 jogadores e 4 vs. 5 jogadores, além de um coletivo com 11 vs. 11 jogadores em campo com medida padrão. As sessões de jogos reduzidos foram divididas em duas etapas com 5 minutos de duração, totalizando 10 minutos e um intervalo de descanso entre elas de 4 minutos, com exceção para o coletivo. O coletivo foi realizado para que fosse feito controle e a comparação entre a situação de igualdade numérica e a inferioridade numérica (3 vs. 4 e 4 vs. 5), observando o comportamento das variáveis do estudo nessas situações. As variáveis analisadas nos jogos reduzidos ( 3 vs. 4 e 4 vs.5) foram apenas dos indivíduos em inferioridade numérica, sendo desconsiderado a superioridade. Os atletas avaliados no coletivo foram apenas do time que participou dos jogos reduzidos ${ }^{3}$.

\section{Análise estatística}

O delineamento da pesquisa determinou a influência do jogo reduzido no teste de atenção concentrada, frequência cardíaca média, frequência cardíaca máxima e percepção de esforço. Os dados da pesquisa foram analisados quantitativamente por meio de técnicas estatísticas descritivas (média e desvio padrão). Foi verificado a normalidade dos dados pelo teste Shapiro-Wilk e foi observada a distribuição dos dados por meio do histograma. Como os dados eram paramétricos foi utilizado o teste ANOVA para comparação dos resultados dos grupos, utilizando-se o teste de Tukey para comparação entre grupos. O tratamento estatístico foi realizado no programa Statistical Package for the Social Sciences (SPSS) versão 20.0 para Windows (Chicago, IL. USA) e o nível de significância estabelecido para todas as situações foi de $p<0,05$.

\section{RESULTADOS}

O estudo comparou os efeitos dos jogos reduzidos em inferioridade numérica sobre as variáveis frequência cardíaca, percepção subjetiva de esforço e atenção concentrada. Na Tabela 1 estão descritas as características do grupo pesquisado. São apresentados as médias e o desvio padrão (DP) da amostra.

Quando comparamos as variáveis com os métodos de treinamento de jogos reduzidos em inferioridade numérica (3 vs. 4 e 4 vs. 5) e coletivo observamos que a frequência cardíaca média $(p<0,121)$, máxima $(p<0,404)$ e a percepção de esforço $(p<0,311)$ não demonstram diferenças entre os métodos de treinamento, conforme Tabela 2. 
Podemos observar na Tabela 3 que os métodos de treino apresentam diferenças no teste de atenção concentrada $(p<0,004)$. Quando realizado o teste de Tukey, comparação entre grupos, podemos observar que o grupo de 3 vs. 4 com o 4 vs. 5 apresenta diferença no teste de atenção concentrada $(p<0,04)$ e também houve diferença entre o 3 vs. 4 e o coletivo $(p<0,033)$. Quando comparamos o 4 vs. 5 com o coletivo não observamos diferença no teste de atenção concentrada $(p<0,678)$.

Tabela 1. Características da amostra.

\begin{tabular}{|c|c|}
\hline Variáveis & Média \pm DP \\
\hline Idade (anos) & $19,1 \pm 2,5$ \\
\hline Massa (kg) & $66,4 \pm 9,9$ \\
\hline Altura $(\mathrm{cm})$ & $175,4 \pm 8,6$ \\
\hline $\operatorname{IMC}\left(\mathrm{kg} / \mathrm{m}^{2}\right)$ & $21,6 \pm 2,4$ \\
\hline Relação Cintura e Quadril & $0,86 \pm 0,05$ \\
\hline Percentual de Gordura & $8,4 \pm 4,5$ \\
\hline Massa Magra (kg) & $60,4 \pm 7,0$ \\
\hline Massa Gorda (kg) & $5,9 \pm 4,1$ \\
\hline Tórax (cm) & $85,4 \pm 6,5$ \\
\hline Abdômen (cm) & $76,3 \pm 6,5$ \\
\hline Antebraço esquerdo (cm) & $25,4 \pm 1,5$ \\
\hline Antebraço direito (cm) & $25,5 \pm 1,5$ \\
\hline Braço Esquerdo (cm) & $30,3 \pm 3,0$ \\
\hline Braço Direito $(\mathrm{cm})$ & $30,1 \pm 3,2$ \\
\hline Cintura (cm) & $75,2 \pm 6,0$ \\
\hline Quadril (cm) & $86,6 \pm 5,1$ \\
\hline Coxa Direita $(\mathrm{cm})$ & $86,6 \pm 5,1$ \\
\hline Coxa Esquerda (cm) & $54,0 \pm 4,4$ \\
\hline Panturrilha Esquerda (cm) & $35,6 \pm 1,9$ \\
\hline Panturrilha Direita $(\mathrm{cm})$ & $35,5 \pm 1,8$ \\
\hline
\end{tabular}

Tabela 2. Comparação das variáveis entre os métodos de treino.

\begin{tabular}{|c|c|c|c|}
\hline Variável & $\begin{array}{c}\text { Métodos de } \\
\text { treino }\end{array}$ & Média \pm DP & $p<0,05$ \\
\hline \multirow{3}{*}{$\begin{array}{c}\text { Frequência } \\
\text { cardíaca média } \\
\text { (bpm) }\end{array}$} & $\begin{array}{c}3 \text { vs. } 4 \\
\text { inferioridade. }\end{array}$ & $160,4 \pm 15,4$ & \multirow{3}{*}{0,121} \\
\hline & 4 vs. 5 inferioridade & $152,4 \pm 8,0$ & \\
\hline & Coletivo & $150,1 \pm 9,2$ & \\
\hline \multirow{3}{*}{$\begin{array}{c}\text { Frequência } \\
\text { cardíaca máxima } \\
\text { (bpm) }\end{array}$} & $\begin{array}{c}3 \text { vs. } 4 \\
\text { inferioridade. }\end{array}$ & $199,0 \pm 18,7$ & \multirow{3}{*}{0,404} \\
\hline & 4 vs. 5 inferioridade & $198,4 \pm 21,2$ & \\
\hline & Coletivo & $189,7 \pm 7,6$ & \\
\hline \multirow{3}{*}{$\begin{array}{l}\text { Percepção } \\
\text { de esforço }\end{array}$} & $\begin{array}{c}3 \text { vs. } 4 \\
\text { inferioridade. }\end{array}$ & $4,7 \pm 1,1$ & \multirow{3}{*}{0,311} \\
\hline & 4 vs. 5 inferioridade & $5,2 \pm 1,9$ & \\
\hline & Coletivo & $4,2 \pm 0,9$ & \\
\hline
\end{tabular}

Tabela 3. Comparação do Teste de Atenção Concentrada entre os métodos de treino.

\begin{tabular}{c|c|c|c}
\hline Variáveis & Média \pm DP & $\%$ & $\boldsymbol{p}<0,05$ \\
\hline 3 vs. 4 inferioridade & $51,9 \pm 28,9$ & $35,2 \pm 19,6$ & \multirow{2}{*}{$0,004^{*}$} \\
\hline 4 vs. 5 inferioridade & $97,2 \pm 27,3$ & $66,1 \pm 18,5$ & \\
\hline Coletivo & $86,3 \pm 30,0$ & $58,7 \pm 20,4$ & \\
\hline
\end{tabular}

* Apresenta diferença significativa.

\section{DISCUSSÃO}

O estudo analisou os efeitos dos jogos reduzidos em inferioridade numérica na frequência cardíaca média, máxima, na percepção de esforço e no teste de atenção concentrada. Apesar de se verificar uma tendência de aumento da frequência cardíaca média e máxima nos jogos reduzidos, a elevação não foi suficiente para que a alteração fosse significante. Impellizzeri et al. ${ }^{8}$, em um estudo longitudinal utilizando os jogos reduzidos em igualdade numérica com 4 vs. 4 e 5 vs. 5 jogadores, com dimensões de campo semelhantes às nossas, apresentou resultados análogos ao do nosso estudo após 12 semanas de intervenção, a frequência cardíaca máxima também não apresentou diferença significativa. Já no estudo de Pasquarelli et al. ${ }^{9}$, os jogadores foram divididos em grupos de 3 vs. 4, 4 vs. 5 e 5 vs. 6, com dimensões de campo superiores às nossas, observou-se uma melhora no condicionamento físico dos atletas, em especial os que treinavam em inferioridade numérica. Embora saibamos que os jogos reduzidos podem elevar a frequência cardíaca em decorrência da manipulação do número de jogadores, a intensidade do jogo parece ser mais afetada quando se altera as dimensões do campo, pois exige dos atletas a necessidade de uma maior movimentação. Contudo, o monitoramento da frequência cardíaca pode subestimar atividades de curta duração e alta intensidade, como os jogos reduzidos, pois a resposta da frequência cardíaca para mudanças bruscas de intensidade não são imediatas ${ }^{9}$.

A percepção subjetiva de esforço é fortemente indicada pela literatura como um método subjetivo e eficiente na monitorização da intensidade do treinamento. Sua correlação com a frequência cardíaca, volume de oxigênio e com as concentrações de lactato apontam dados mais confiáveis a respeito da carga de treino, além de ser um método não invasivo e de baixo custo $^{2}$. Em nosso estudo, a percepção subjetiva de esforço não apresentou diferença significativa. Isso pode ser explicado pela correlação existente entre a frequência cardíaca e a percepção subjetiva de esforço, e uma vez que a frequência cardíaca não apresentou diferença significativa, era esperado que a percepção subjetiva de esforço também não apresentasse ${ }^{8}$. Segundo Little e Willians ${ }^{21}$, a percepção subjetiva de esforço apresentou diferença significativa quando trabalhada com um número reduzido de jogadores (2 vs. 2), além disso, os níveis de lactato sanguíneo também estavam elevados, o que corrobora com resposta subjetiva dos atletas. Sendo assim, um método de treinamento com um número reduzido de jogadores pode enfatizar o desenvolvimento das vias anaeróbias, exigindo uma maior intensidade dos atletas.

No nosso estudo os testes de atenção concentrada apresentaram diferença significativa nos jogadores em inferioridade numérica, elevando a capacidade de atenção dos atletas pós-treino. Isto se explica pela necessidade de suprir a falta de um determinado jogador, fazendo com que os atletas trabalhem sobre pressão e elevem seu nível de atenção durante os treinamentos ${ }^{13}$. A atenção concentrada é a capacidade de concentrar e executar uma atividade, sendo importante durante o jogo. $\mathrm{O}$ atleta que apresenta bons resultados na avaliação da atenção concentrada demonstra bom controle cognitivo e mental e responde melhor a situações de pressão e estresse ${ }^{13}$. Os atletas que atuam na defesa durante o jogo trabalham em situações de pressão e com grau de concentração elevada demonstrando resultados melhores no teste de atenção ${ }^{13,22}$. Segundo Esturillo ${ }^{13}$ a atenção é uma habilidade cognitiva que está associada às capacidades físicas dos atletas de futebol. A atenção pode ser desenvolvida e treinada e pode sofrer efeitos do tempo e método de treinamento. Acreditamos que os jogos reduzidos em inferioridade numérica podem contribuir para melhora da atenção concentrada nos atletas diminuindo as falhas principalmente ao final do jogo. Ao final de uma partida a incidência de gols é maior em decorrência do cansaço e da baixa concentração. Portanto, podemos observar que 
há uma correlação entre as variáveis psicológicas e o cansaço ao final do jogo influenciando no resultado da partida 23,24 .

\section{CONCLUSÃO}

O estudo comparou os efeitos do treinamento de jogos reduzidos no teste de atenção concentrada, frequência cardíaca e percepção de esforço no futebol. Concluímos que os jogos reduzidos com inferioridade numérica 3 vs. 4, 4 vs. 5 e coletivo melhoram os resultados do teste de atenção concentrada. Já na frequência cardíaca e percepção subjetiva de esforço não apresentaram diferenças significantes.
Sugerimos para os próximos estudos dimensões menores de campo que pode influenciar na intensidade do jogo reduzido.

\section{AGRADECIMENTOS}

Os autores agradecem à Fundação de Amparo à Pesquisa do Estado de Minas Gerais - FAPEMIG e Centro Universitário de Itajubá - FEPI.

Todos os autores declararam não haver qualquer potencial conflito de interesses referente a este artigo.

CONTRIBUIÇÕES DOS AUTORES: Cada autor contribuiu individual e significativamente para o desenvolvimento do manuscrito. VHSM (0000-0003-0758-3299)* elaborou do projeto de pesquisa, escrita do manuscrito, execução dos programas de treinamento e coleta de dados. GPM (0000-0002-4899-7204)* contribuiu com a escrita do manuscrito, execução dos programas de treinamento, coleta e análise dos dados. ASS (0000-0002-9603-7881)* participou no desenho do estudo, elaboração do projeto de pesquisa, análise estatística, revisão e aprovação final da versão do manuscrito. FVL (0000-0002-0617-2423)* contribuiu no desenho do estudo, elaboração do projeto de pesquisa e aprovação do manuscrito. JAOS (0000-0002-5782-2043)* contribuiu no desenho do estudo, elaboração do projeto de pesquisa, aprovação do manuscrito, coleta e análise dos dados. RJB (0000-0003-4828-5212)* e LHSO (0000-0002-3994-2316)* realizaram a revisão crítica do manuscrito, conteúdo intelectual e aprovação final da versão do manuscrito. ${ }^{*} \mathrm{ORCID}$ (Open Researcher and Contributor ID).

\section{REFERÊNCIAS}

1. Dellal A, Lago-Penas C, Wong DP, Chamari K. Effect of the number of ball contacts within bouts of 4 vs. 4 small-sided soccer games. Int J Sports Physiol Perform 2011;6(3):322-33.

2. Alexiou H, Coutts AJ. Comparison of methods used for quantifying internal training load in women soccer players. Int J Sports Physiol Perform. 2008;3(3): 320-30.

3. Hill-Haas S, Rowsell G, Coutts A, Dawson B. The reproducibility of physiological responses and performance profiles of youth soccer players in small-sided games. Int J Sports Physiol Perform. 2008;3(3): 393-6.

4. Katis A, Kellis E. Effects of small-sided games on physical conditioning and performance in young soccer players. J Sports Sci Med. 2009;8(3): 374-80.

5. Praça GM, Silva MV, Greco PJ. Manipulações no tamanho do campo e número de jogadores em pequenos jogos no futebol-Revisão sistemática. Acta Bras Mov Hum. 2015;4(5):47-72.

6. Dellal A, Hill-Haas S, Lago-Penas C, Chamari K. Small-sided games in soccer: amateur vs. professional players' physiological responses, physical, and technical activities. J Strength Cond Res 2011;25(9): 2371-2381.

7. Aguiar MVD, Botelho GM, Gonçalves BS, Sampaio JE. Physiological responses and activity profiles of football small-sided games. J Strength Cond Res. 2013;27(5): 1287-94.

8. Impellizzeri FM, Marcora SM, Castagna C, Reilly T, Sassi A, laia FM, et al. Physiological and performance effects of generic versus specific aerobic training in soccer players. Int J Sports Med. 2006;27(6): 483-92.

9. Pasquarelli BN, Souza VAFA, Stanganelli LCR. Os jogos com campo reduzido no futebol. Rev Bras Futebol. 2010;3(2): 2-27.

10. Aslan A. Cardiovascular responses, perceived exertion and technical actions during small-sided recreational soccer: effects of pitch size and number of players. J Hum Kinet. 2013;38,95-105.

11. Castellano J, Casamichana D, Dellal A. Influence of game format and number of players on heart rate responses and physical demands in small-sided soccer games J Strength Cond Res. 2013;27(5):1295-303.

12. Sampaio JE, Lago C, Gonçalves B, Maçãs VM, Leite N. Effects of pacing, status and unbalance in time motion variables, heart rate and tactical behaviour when playing 5 -a-side football small-sided games.
J Sci Med Sport. 2014;17(2):229-33

13. Esturillo RC. Estudio exploratorio de la prueba perceptiva y de atención Toulouse - Piéron en jugadores de fútbol. Lecturas de Educación Física y Deportes. 2002;8(46).

14. Fonseca PHS, Marins JCB, Silva AT. Validação de equações antropométricas que estimam a densidade corporal em atletas profissionais de futebol. Rev Bras Med Esporte. 2007;13(3):153-6.

15. Araujo Jr JH, Marques RF, Costa HA, Marques KRM, Almeida RB, Oliveira Jr MNS. Comparação do teste de Rast em jogadores de futebol e futsal de nível universitário. RBPFEX. 2012;6(34): 367-71.

16. Mcmillan K, Helgerud J, Macdonald R, Hoff J. Physiological adaptations to soccer specific endurance training in professional youth soccer players. Br J Sports Med 2005;39(5): 273-7.

17. Coelho DB, Mortimer LACF, Condessa LA, Soares DD, Barros CLM, Garcia ES. Limiar anaeróbio de jogadores de futebol de diferentes categorias. Rev bras cineantropom. Desempenho Hum. 2009;11(1):81-7.

18. Duarte R, Batalha N, Folgado H, Sampaio J. Effects of exercise duration and number of players in heart rate responses and technical skills during futsal small-sided games. Open Sports Sci J. 2009;2:37-41.

19. Borg GA. Psychophysical base of perceived exertion. Med Sci Sports Exerc. 1982;14(5):377-81.

20. Cecilio-Fernandes D, Rueda FJM. Evidência de validade concorrente para o Teste de Atenção Concentrada (TEACO-FF). PSIC. 2007:8(2):167-74

21. Little T, Williams AG. Measures of exercise intensity during soccer training drills with professional soccer players J Strength Cond Res 2007;21(2):367-71

22. Mahl AC, Raposo JV. Perfil psicológico de prestação de jogadores profissionais de futebol do Brasil. Rev Port Ciênc Desp. 2007;7(1):80-91

23. Zacarias F, Olivas MA, Silva AS. Incidência de gols nas partidas de futebol de salão nos jogos escolares de Minas Gerais no módulo 1 da fase municipal de Itajubá. RBFF. 2015;7(23):21-4.

24. Santos TCB. A incidência de gols na fase classificatória para a copa do mundo de 2010 na África do Sul. RBFF. 2015;7(23):67-71. 